\title{
CULTURE ET CULTURAL STUDIES
}

\author{
Andrea Catellani ${ }^{1}$
}

Lire un texte de Jan Baetens est toujours une expérience très satisfaisante : l'article dédié à la culture et à sa définition liée aux Cultural Studies (études culturelles) ne fait pas exception. Ce qui frappe, outre la clarté stylistique - qui fait penser à une sorte de limpidité classique, opposée à certains styles baroques quelque fois présents dans le milieu intellectuel européen - est l'abondance de références tant traditionnelles que contemporaines, et le conséquent panoptisme heureux démontré par l'auteur. Cette limpidité se lie à la profondeur théorique, toujours avec un sens de légèreté qui - à en croire les American Lectures d'Italo Calvino - reste une des valeurs centrales pour notre temps. Jan Baetens veut montrer, entre autres, comment les Cultural Studies (dorénavant, $\mathrm{CS}$, au masculin) ont aidé à la récupération de l'expérience de la beauté et du plaisir esthétique contre une certaine dérive autoréférentielle et anti-esthétique de l'art contemporain : nous pouvons affirmer que cette récupération passe du contenu à l'expression de son texte, du sujet traité au style discursif et à la façon de construire le discours.

Un autre aspect que nous avons apprécié est la capacité de proposer des oppositions sans caricaturer les termes opposés : par exemple, la présentation de la position " élitiste » de la culture, propre à Matthew Arnold, ne se réduit pas à une esquisse ridicule facile à critiquer et à dépasser, mais introduit ses aspects pertinents et stimulants par rapport à ce qui a été fait après Arnold. Le résultat de cette démarche est une

1 Andrea Catellani est professeur au département de communication de l'UCL. 
forme de problématisation qui cherche à restituer tout leur poids aux différentes positions, tout en défendant une certaine thèse.

Nous avons décidé de suivre le discours de Jan Baetens sur la culture et les CS presque pas à pas, pour trouver ici et là des occasions pour quelques modestes gloses. Ainsi faisant, nous trouverons grosso modo trois grands sujets à traiter : la culture, les CS comme méthodologie et tradition dotée d'une histoire riche, enfin les relations de cette approche avec différentes disciplines, en particulier, l'histoire culturelle et la (les) sémiotique(s).

La première partie de l'article de Baetens est dialectique/apologétique : l'auteur présente la complexité du concept de « culture » et une des approches face à cette complexité, celle des CS. L'auteur cherche alors à mettre en évidence les particularités des CS, les critiques qu'ils reçoivent et les réponses à ces critiques. Cette partie apparaît très utile pour dégager les traits communs des CS. Dans la deuxième partie, l'approche ainsi proposée est mise à l'épreuve d'une forme culturelle concrète, celle de la bande dessinée flamande de l'après guerre, pour montrer concrètement pourquoi les CS méritent de faire encore partie du panorama des recherches sur les formes culturelles.

\section{Culture et Cultural Studies}

L'auteur affronte avant tout le problème de la définition de la culture. En effet, il s'agit d'un concept, ou plus concrètement d'un mot, ouvert à toutes les acceptions. Si Umberto Eco, au milieu des années 90, avouait de ne plus savoir ce qu'est un symbole, à cause de la prolifération des définitions et des usages, il sera d'autant plus difficile de dire ce que c'est la (une) culture. Jean-Marie Schaeffer disait récemment (2007) que déjà en 1952 Kluckhon, Kroeber, Meyer et Untereiner avaient répertoriés 150 définitions différentes de culture ; comme conséquence, son choix, en travaillant sur ce concept, était d'en proposer une conception purement instrumentale, une notion opératoire et non-essentialiste. En réalité, cette distinction entre « essence » et « notion opératoire » ne l'empêche pas de proposer une liste des différents types ou « classes fonctionnelles » de la culture ${ }^{1}$, et d'arriver à l'affirmation globale que les « faits culturels» forment une « ontologie distribuée » (p. 281). Le choix de Jan Baetens est différent, tout en n'étant pas alternative. Il

1 Matériel, social, agentif, institutionnel, normatif, symbolique. Schaeffer 2007, p. 276. 
laisse en effet le champ ouvert, et introduit seulement une théorie de la culture traditionnelle (celle d'Arnold) et une proposition alternative, celle des CS : le problème de la définition de la culture est déplacé et devient une tentative de définir ses limites et la forme que prend sa configuration catégorielle. L'opposition choisie est celle, bien connue, entre une conception restreinte, élitiste, de la Culture comme Bildung, sommet des productions humaines dignes d'être transmises, et l'ouverture « démocratique » permise par les CS envers toutes les formes et pratiques culturelles qui forment une civilisation déterminée. De ce point de vue, on trouve au fond l'opposition entre deux différents styles de valorisation, identifiés par la sémiotique tensive française ${ }^{1}$ : celui des « valeurs d'absolu » (valorisation faite à travers la sélection du mieux, des « happy few ») et celui des « valeurs d'univers » (valorisation sur la base de l'extension maximale). Mais le discours de Jan Baetens est plus complexe que ça, parce qu'il nuance et situe dans une perspective historique et contextuelle les deux positions. Par rapport à la théorie « culturaliste » de Matthew Arnold, qui mêle illuminisme et romantisme, Jan Baetens souligne que la « mauvaise presse » de cette conception est justifiée seulement en partie et que d'autres lectures sont possibles. Du côté des CS, il affirme par contre qu'il s'agit d'une contribution qui se veut modeste et complémentaire aux autres approches de la culture. Il ne s'agit pas non plus d'éliminer toutes formes de « tri », de sélection, de jugement ni d'obtenir un aplatissement complet : Baetens rappelle que les CS n'éliminent pas la possibilité du jugement de valeurs artistique, mais le déplacent à l'intérieur de chaque forme culturelle (théâtre, bande dessinée, etc.). Le principe unifiant de la hiérarchie n'est pas éliminé, mais pluralisé : chaque monde culturel, médiatique, artistique aura alors ses médiocrités et ses excellences (des bons et des mauvaises Shakespeare, des bons et des mauvaises Tin Tin).

La stratégie de l'auteur apparaît alors clairement : éliminer les oppositions faciles, idéologiques, mettre en évidence les superstructures connotatives (la définition élitiste de la culture comme bouc émissaire) pour rouvrir le discours et montrer la complexité du réel. En reprenant certaines catégories de Anne Beyaert-Geslin et Jacques Fontanille (2003), il s'agit d'un mouvement relativisant proche de la « conversion méta-textuelle », qui transforme ce qui se veut connotatif, « naturel », absolu et sans alternatives en une possibilité de lecture

1 Voir Fontanille et Zilberberg 1998. 
parmi d'autres (une variation possible dans un paradigme). Ce travail de problématisation et de mise en perspective s'harmonise parfaitement avec l'approche anti-idéologique et critique que les CS veulent adopter envers les formes culturelles. Le niveau supplémentaire apporté par Jan Baetens est l'application de cette dynamique à l'origine même des CS et à leur propre démarche, et donc un mouvement de réflexivité. Le résultat est une lecture différente et ouverte de l'origine polémique des CS et de leur « adversaire ».

\section{Cultural Studies : définitions et caractères}

Pouvons-nous donner une définition des CS ? La réponse de l'auteur est négative : tout ce que l'on peut faire est chercher « quelques traits communs » (p. 2), capable de relier entre eux les membres d'une pluralité irréductible. On pourrait alors se demander quel «style de catégorisation » permettrait de saisir cette pluralité. L'approche choisie par l'auteur est la définition d'une série de traits nécessaires, regroupés selon les catégories de l'objet, de la méthode et de la visée des CS ${ }^{1}$. Ces traits forment des frontières communes, un « dedans » et un " dehors ", et laissent exister à l'intérieur de l'ensemble ainsi défini un nombre illimité de variations et différences.

Pour ce qui concerne la « culture » comme objet formel des CS, Baetens souligne en particulier deux moments, le début et la situation actuelle : la définition initiale, donnée par Hoggart et les autres pionniers, de la culture comme « way of life » et comme totalité des éléments d'une civilisation, d'un point de vue plutôt anthropologique ; et l'approche de plus en plus pragmatique, influencée par le milieu américain en particulier, qui a triomphé par la suite. De ce point de vue, on retrouve un des grands tournants de la culture intellectuelle du XX

1 Du point de vue de l'objet, on trouve au centre l'élargissement des formes culturelles pertinentes pour l'analyse, et l'ouverture à une vision pragmatiste et symbolique des faits culturels. Du point de vue de la méthodologie, émerge le refus des clôtures disciplinaires, l'ouverture au contexte, la tentative de lier macro et micro et l'implication personnelle du chercheur, qui est aussi militant. Enfin, du point de vue de la visée des CS, il est nécessaire de poser la considération de la culture comme instrument de pouvoir, force réelle jamais neutre ou inefficace, et donc, dans une « vision maximaliste » des CS, l'idée que le chercheur doit intervenir directement, « sortir de la culture, pour faire autre chose, en l'occurrence de la politique » (p. 5). Cette liste définit le champ à l'intérieur duquel on trouve des « airs de famille », des liens multiples et partiels entre différentes tendances et formes en évolution. 
siècle, celui qui à porté à valoriser de plus en plus l'expérience concrète et les pratiques de la subjectivité. Cette évolution a adopté différentes formes, entre autres en philosophie (on pense à Bergson et à la phénoménologie), en linguistique et en sémiotique ${ }^{1}$, et en sociologie. Les CS s'insèrent dans cet ensemble, vivent à leur tour ce passage et ce tournant pragmatiste (et « contextualiste »).

Mais nous pouvons évidemment ajouter à ce grand tournant (culture comme Way of life vs. culture comme expérience et expérimentation, comme ensemble de pratiques en devenir) d'autres ruptures, et d'autres passages, pour compléter l'histoire des CS. En particulier, l'influence profonde du structuralisme, plus ou moins lié au marxisme et à sa relecture, par exemple dans le cas de Althusser et de sa conception du « sujet interpellé » et assujetti, mais aussi dans celui des travaux de Lévi-Strauss, Barthes, Foucault, Lacan, et Antonio Gramsci. Entre la première partie « culturaliste » de Hoggart, Thomson et Williams, et l'explosion internationale (et américaine surtout) à dominance " pragmatiste », il existe donc toute une phase « structuraliste » et « anti-idéologique » des CS, favorisée en particulier par la présence de Stuart Hall à la direction du Centre for Contemporary Cultural Studies (CCCS) de Birmingham, entre 1969 et 1979. Il ne faut pas oublier non plus la relecture des théories de l'italien Gramsci, comme celle de la relation entre intellectuels traditionnels et organiques et, surtout, le concept centrale d'hégémonie. Cette contribution a été d'une importance capitale pour les CS, et pour leur façon de comprendre la culture populaire comme lieu de tension et d'échange entre projets hégémoniques des groupes dominants et formes de résistance des groupes subordonnés.

Comme le soulignait Roberto Grandi, en résumant l'histoire des CS (1994, p. 104), cette phase structuraliste contribua à « injecter» dans les CS les apports de la sémiotique. Celle-ci a transmis aux CS les effets $\mathrm{du}$ 《 tournant linguistique » qui, entre autres choses, a imposé pour plusieurs années le texte et, ensuite, la textualité comme lieu central de l'analyse et comme dispositif puissant d'imposition idéologique. Le déterminisme textuel, protagoniste de la recherche des années 70, laissa la place, pendant la décennie successive, à une attention grandissante pour le contexte, pour les formes de résistance, pour les subcultures : ce sont les années des analyses ethnographiques sur les usages sociaux

1 Avec le passage du premier au deuxième Wittgenstein, les études sur le langage ordinaire, l'énonciation, les actes de langage et la dynamique de l'interprétation avec Peirce et Eco, et le développement d'une sémiotique des pratiques. 
de la télévision, proposées par James Lull. Ce virage bien connu vers une conception moins omnipuissante du texte et vers une attention plus grande pour le lecteur et pour le contexte a également été caractérisé par l'apparition, toujours à partir de la fin des années 70 , des recherches sur la postmodernité, les problématiques de gender, l'approche postcolonial, et encore par l'influence d'autres perspectives de recherche comme le New Historicism, l'anthropologie interprétative, les recherches sur la construction de la subjectivité. Jusqu'aux critiques faites, vers la fin des années 80 , à certaines dérives excessivement anti-textualiste, avec les dénonciations des risques de perdre la possibilité d'un discours critique et de se rendre à la culture postmoderniste dominante ${ }^{1}$. Il ne faut pas oublier que, en sémiotique, ce sont les années de la dénonciation des dérives déconstructionnistes et " pragmatistes », faite par Eco (1990/1999) en rappelant les limites textuels du pouvoir de création du sens.

Une petite histoire, très partielle, des CS permet, on le voit, de pénétrer au cœur des évolutions fondamentales de certains sciences humaines occidentales des dernières décennies, une évolution faite souvent de ruptures successives.

Sur le plan méthodologique, Jan Baetens reconnait les limites implicites de(s) approche(s) des CS. L'interdisciplinarité est «plus facile à poser en théorie qu'à réaliser en pratique » (p. 4), et ceci au-delà de la rhétorique dominante aujourd'hui dans les milieux académiques. L'interdisciplinarité des CS est liée à sa nature de projet critique et de changement politique, mené par des intellectuels qui se veulent souvent organiques (aux subcultures, aux classes dominées, contre les cultures dominantes), et qui mobilisent chaque fois les outils meilleurs pour comprendre les objets et pour pouvoir influencer la réalité. Les problèmes de " fragilité » scientifique des CS, reconnus par Jan sont, apparaissent en tout cas communs à d'autres projets de recherche, comme peut-être celui de l'analyse culturel proposée par Mieke Bal ou les propositions d'une science de la communication comme « interdiscipline ». Les CS ont indubitablement aidé la diffusion du « mot de passe » de l'interdisciplinarité, en contribuant à l'évolution épistémique contemporaine des sciences humaines. La tension entre nécessaire ouverture interdisciplinaire et nécessité de garder la rigueur et la capa-

1 Culture postmoderniste reconnue déjà par Jameson en 1991 comme logique culturelle du capitalisme tardif, comme le souligne Jan Baetens. 
cité euristique (et d'éviter la confusion) est un des enjeux des sciences sociales et culturelles aujourd'hui.

Une spécificité centrale des CS, comme le dit l'auteur, réside dans leurs visées et dans leurs enjeux. De ce point de vue, reste toute la difficulté d'un choix spécifique des CS, celui de l'engagement qui « dépasse l'observation participante que l'on recommande dans d'autres disciplines » (p. 4), et donc d'une position qui « court-circuite » la distinction traditionnelle entre moment d'analyse et moment d'intervention politique. La tradition spirituelle nous enseigne que certains types de connaissance sont fondés sur l'amour : les CS semblent proposer une version « moderne » de ce constat. Le point est délicat, et nous trouvons qu'il faut poser quelques distinctions à ce propos.

La construction du savoir se fonde traditionnellement sur la distinction théorique entre rôles actanciels différents du même acteur : il faut distinguer le rôle de chercheur et celui d'agent actif de changement culturel et politique. La même personne peut avoir ces deux rôles sans nécessairement confondre les plans. Cette distinction théorique permet alors différentes positions, dont celle des membres « maximalistes » des CS, qui pourront par exemple intervenir directement en politique et pratiquer une quelque forme de ce que Eco appelait jadis « guérilla sémiologique », pendant que d'autres se limiterons à exposer une certaine modélisation. Mais, pour défendre les CS, Jan Baetens affirme que la combinaison de scientificité et militantisme est fondée sur la nécessaire relation entre fait culturel et interprétation, et que donc la neutralité axiologique est impossible « tant qu'on reste à l'intérieur du champ [culturel] » (p. 5). La distinction entre être et ne pas être dans un champ culturel serait à fonder et travailler, mais nous nous contentons de rappeler quelques uns des termes du problème ainsi évoqué, et qui est assez traditionnel en sciences sociales. Est-ce que nous pouvons imaginer des formes de recherche qui restent pénétrantes (capables de comprendre de l'intérieur certains faits culturels) tout en s'efforçant de ne pas suivre une position axiologique précise ? Est-ce que la recherche culturelle implique nécessairement une prise de position axiologique, la construction d'un vouloir faire (vouloir défendre ou attaquer la forme culturelle), fondement immédiat d'une action militante ? Nous pensons que plusieurs positions doivent rester parcourables (ce qui, en tout cas, n'est pas nié par l'auteur), et que les options affichées d'engagement subjectif et de discipline neutralisante doivent être jugées par leurs fruits. Les deux positions extrêmes du continuum, la neutralité la plus rigoureuse et l'engagement le plus militant, peuvent, toutes deux, 
faire obstacle à la connaissance en empêchant à la réalité culturelle de s'exprimer au delà des préjugés et des schémas de l'observateur. Une coexistence trop immédiate d'observation et de militantisme peut par exemple forcer les résultats, alors qu'une certaine recherche (nous dirions " modérée ») de neutralité axiologique, entendue comme choix tactique et comme position momentanée, pourrait paradoxalement mieux fonder l'engagement successif, tout en sachant que la neutralité absolue reste un idéaltype.

Le débat est en tout cas destiné à rester ouvert, et nous trouvons très judicieuse l'observation finale de Jan Baetens qui affirme la nécessité d'un effort de "vérification intersubjective », qui doit ouvrir les résultats de chaque approche méthodologique aux autres.

\section{Faire des cultural studies aujourd'hui : la défense}

La défense faite par l'auteur de l'importance des CS aujourd'hui est intelligente et convaincante, focalisée en particulier sur le versant de l'art et de la pensée esthétique. Les CS ont été accusés d'empêcher le jugement esthétique, de politiser l'approche à l'art, d'opérer un nivellement vers le bas des formes artistiques et d'accélérer la « commodification » des formes artistiques en contribuant à la commercialisation de la culture. Jan Baetens réplique point par point, pour éviter la réduction des CS à un bouc émissaire de phénomènes bien plus généraux. Sur le point de la crise du jugement esthétique, Jan Baetens admet entre autres le populisme des CS comme exaltation du «populaire » en tant que tel, mais rappelle que c'est une «maladie infantile » dépassée aujourd'hui, et non généralisée. Pluraliser et contextualiser le canon esthétique et culturel ne veut pas dire éliminer le jugement de qualité, mais s'ouvrir à la pluralité des formes culturelles - même si, il faut l'admettre, l'augmentation de la complexité rend plus difficile, " coûteux » et incertain le jugement, et aussi, sur d'autres plans, plus difficile la recherche du lien social et de l'unité autour de certaines formes culturelles. C'est le prix cognitif et social de la complexité, qui génère le malaise actuelle à synthétiser (sans réductionnismes et totalitarismes) sur le plan esthétique, social et même politique. Mais ces problèmes, qui appartiennent à la notre contemporanéité, ne doivent pas empêcher de voir l'importance des ouvertures, du regard aux contextes, aux « coulisses » culturelles et à la dimension politique, permises par les CS. La stratégie défensive de Jan Baetens est correcte : placer les CS comme complément important des recherches et des approches à la culture, voix entre autres dans le 
concert pluriel de la recherche, toujours soumise au contrôle intersubjectif.

Nous trouvons urgent et justifié l'accent mis par l'auteur sur l'importance de l'étude et de la critique des infatigables mécanismes de reprise « populiste », par le système capitaliste tardif, des formes culturelles. Les CS n'empêcheraient pas la recherche d'une remontée du 《 culturel » vers la « culture » et même vers le « culte », pour reprendre la trichotomie historique proposée par Jean Clair et justement citée par Baetens. Ils rendent seulement cette recherche de la qualité et de la valeur plus consciente des différents niveaux impliqués, plus « responsable », plus avertie et donc plus exigeante, mais aussi plus adulte et complète.

L'auteur n'oublie pas de montrer que l'équation culture/politique (et donc, l'excessive politisation de la culture) n'est ni nécessaire ni naturelle, mais contingente, et le but des CS peut être le dépassement de cette forme généralisée d'instrumentalisation du fait culturel. L'utopie n'est pas absente de cette perspective, l'utopie, à mieux préciser, d'une humanité libérée aussi de ce point de vue. Reste ouvert, selon nous, le problème du contenu concret de la visée politique et utopique des $\mathrm{CS}$, pour ne pas tomber dans les dangers du relativisme, de la défense de n'importe quelle forme culturelle et de la reprise paradoxale d'une sorte d'absolutisme à l'envers (défense d'une certaine forme culturelle en tant que, par exemple, la vraie forme « populaire », avec des effets paradoxales d'exclusion d'autres formes).

Sur le plan méthodologique Baetens propose, on l'a vu, une forme de complémentarisme intelligent : l'attention portée à la relation culture/ politique n'est pas exclusive ni réductrice, les mécanismes de production et d'évolution du sens sont pluridimensionnels, il faut laisser aux faits culturels toute leur richesse interne pour les comprendre, et donc ne pas retomber dans des formes (par exemple, marxistes) de réductionnisme. Nous ne saurions dire ici si l'auteur parle de la situation aujourd'hui, ou plutôt d'un projet, d'un désir ; la lutte contre les réductionnismes, et en même temps contre la perte d'emprise sur la réalité (par excès de complexité), est continuelle. De l'autre côté, un danger toujours présent est l'excessive «fluidité théorique » des CS dans leur version globalisée et triomphante actuelle, dénoncée déjà au début des années 80 par Stuart Hall (cit. in Grandi 1994, p. 108). L'excessive académisation et le succès des CS conduisent à multiplier les discours sur la relation entre pouvoir et culture, tout en perdant une efficacité réelle extra-académique, une possibilité d'opérer le changement : la 
possible auto-référentialité des CS, dans un contexte de « tribalisation » et fragmentation de la culture en général, met en danger le projet politique même de cette approche. Une fois éliminé l'absolutisme excluant, il faut continuer à retravailler le projet des CS, pour l'adapter à une réalité complexe et qui tende en partie à la fragmentation.

Le point central du discours est, comme le souligne l'auteur, la défense des CS contre l'attribution du rôle de destructeurs de l'art, de la culture et du plaisir esthétique. Au contraire, comme le dit Baetens, la remise à la une du plaisir opérée, entre autres, par les CS, permet de contrer sa destruction dans le milieu de l'art avec un grand «A» (p. 8). Sur ce point fondamental, Baetens trouve des alliés puissants, comme Jean-Marie Schaeffer et sa modélisation critique de la « conception transcendantale de l'art ». Tous deux se retrouvent ensemble à contraster cette conception de l'art comme entité monadique auto-référentielle, limitée à peu de formes légitimes, détachée du plaisir, du beau et de toutes finalités et «effets ». Selon les CS et selon Baetens, l'art peut servir à quelque chose tout en restant art, comme c'était normal - nous ajoutons - avant le XVIIIème siècle. Et reste art même si on sort de la dérive autoréférentielle de réflexion sur soi-même et sur ses propres moyens, et si on retrouve le beau et le plaisir. Un autre allié de Baetens, paradoxalement, est la position élitiste de Jean Clair, quand il attaque l'art du déchet et de l'immonde, propre du « culturel» en tant que terme contraposé à la culture. Cet art est un produit d'une évolution du monde même de l'art et de la réflexion esthétique. Ce qui reste à faire, après la clarification et la mise en perspective, c'est peut-être une réflexion sur les relations entre ces deux termes évoqués, le beau et le plaisir, pour distinguer différentes facettes d'un problème éternel pour la philosophie.

\section{La pratique et l'analyse : Cultural Studies, sémiotique et histoire culturelle}

La deuxième partie de l'article de Jan Baetens est un très bel et convaincant exemple d'analyse inspirée par (certains) résultats et approches des CS. L'auteur montre un aspect décisif des CS, c'est-àdire la volonté qu'ils ont de prendre en compte la complexité. Il suffit de relire la liste partielle des aspects pertinentes : « La forme de la bande dessinée, l'histoire, le contexte social, l'interaction entre mots et images, le style et la forme des textes, les allusions culturelles et politiques, le dialogue avec le lecteur, le jeu avec les contraintes insti- 
tutionnelles, le plaisir de raconter (ou de brouiller) un récit...» (p. 10). De ce point de vue " panoptique », la «mauvaise qualité » de la bande dessinée flamande entre la Guerre Mondiale et les années ‘60 n'est pas " seulement » un problème d'incapacité, une réalisation mineure du modèle de la « ligne claire », mais se présente avant tout comme aspect d'une forme culturelle vivante, lieu d'expression d'une réalité sociale et politique. Si on sort du jugement idéologique et formel, on découvre qu'il ne s'agissait même pas de bandes dessinées ${ }^{1}$, mais d'un véritable média populaire. L'ensemble des thèmes et des messages est dans ce contexte décidément plus important que la finition esthétique, et détermine donc la distance par rapport à l'esthétisme des francophones. La « laideur » comme forme de l'expression est strictement liée à la forme du contenu et motivée par elle : comme le souligne depuis toujours la sémiotique (et Baetens est bien conscient de ça, en adoptant le même point de vue), l'organisation de l'expression est nécessairement liée à celle du contenu. A ce propos, le constat de l'« alignement» au style « léché 》 et à des contenus décidément moins violents et « réalistes 》 d'un auteur comme Vandersteen est très frappant : le passage du système dominé (la pseudo-BD des journaux politiques flamands) au système dominant (le journal de Tin Tin) s'exprime au même temps sur le plan de l'expression et sur celui du contenu.

Cette deuxième partie empirique montre comment la sémiotique actuelle, discipline assez malmenée aujourd'hui dans les milieux académiques mais encore active, est capable de nourrir l'approche des CS dans leur version présentée par Jan Baetens. Le référent théorique, proposé pour fonder le passage d'une forme d'analyse limitée au média et au genre à l'ouverture vers la complexité des formes culturelles, est (en partie) sémiotique. Il s'agit en effet du résultat du passage récent (et complexe) de la sémiotique «formelle et formaliste », centrée sur le texte et sur son organisation interne immanente, à une sémiotique de la culture, comme celle proposée par Jurij Lotman et aujourd'hui reprise par des auteurs comme Fontanille en France (2008) et Paolo Fabbri en Italie, surtout du point de vue de l'étude des pratiques. Le passage à la vision des CS « coïncide en partie », « sur le plan théorique » (p. 11), avec ce passage, qui s'accompagne, en sémiotique, d'un passage vers le corps, les passions, la dimension « sensible » du sens. La sémiotique est donc une partie importante de l'arrière-fond théorique des CS (version

$1 \mathrm{Ou}$, comme nous préférerions dire, pas seulement. 
Jan Baetens), une sorte de justification de cette démarche, surtout quand les CS cherchent de « lier macro et micro », comme dit Jan Baetens, en se trouvant en contact direct avec des textes concrets qui sont à mettre en relation avec leur contexte et leur valeur culturelle et sociale. Ce serait intéressant d'étudier les différents aspects des relations entre les CS et le développement de la sémiotique des dernières décennies. Nous avons l'impression, par exemple, que le tournant «pratique» et culturel actuel de cette discipline (au moins dans ses versions plus proche à l'héritage génératif de Greimas) a été favorisé, entre autres, par certaines expériences émergées à l'intérieur des CS. En tout cas, pour plusieurs aspects, les deux mondes ont procédé en parallèle. La sémiotique est, elle aussi, passée d'un modèle structuraliste à une prédominance variée de la pragmatique (ou de l'approche "pragmaticiste» de Peirce). Les travaux de Roland Barthes sur les mythologies contemporaines et ceux d'Eco, suivis des études d'Algirdas-Julien Greimas et de Jean-Marie Floch sur des objets comme la soupe au pistou et le métro parisien, ont contribué à enrichir et ouvrir la recherche sociale. Enfin, la sémiotique, surtout au début, a eu une dimension militante, comme le démontre l'idée d'une "guérilla sémiologique » proposée par Eco dans les années 70. Pour en revenir à Jan Baetens en particulier, sa position est comparable à celle d'autres grands maîtres contemporains qui connaissent et utilisent la sémiotique et ses catégories à l'intérieur d'autres champs disciplinaires, pour les nourrir : nous rappelons seulement Victor Stoichita pour l'histoire de l'art, et Bruno Latour pour la sociologie.

Le dernier lien que nous proposons, en glosant le texte de Jan Baetens, est celui avec l'histoire culturelle. Une analyse comme celle que l'auteur esquisse à propos de la $\mathrm{BD}$ flamande est en partie un travail d'historien culturel. Il faut en effet reconstruire l'époque, sa mentalité, son réseau de pratiques et formes culturelles : il faut contextualiser historiquement le sens. Cet aspect historiciste des CS est partagé par certains historiens qui cherchent à dégager les mentalités et les formes culturelles d'époques passées, souvent avec une approche en partie semblable au CS, ce même au niveau de l'engagement militant. Nous pensons par exemple au récent Sauver le Grand Siècle? de Christian Jouhaud (2007), où le mythe historiographique du siècle de Louis XIV (connoté académiquement et politiquement en France) est déconstruit à partir de l'analyse des mémoires du valet de chambre du Roi Soleil. Ce travail, qui porte ensemble sur les mythes historiographiques actuels et sur les pratiques culturelles d'une époque passée, manifeste une série de « ressemblances de famille» avec les axes de fond des CS. 
Cette série de gloses nous a peut-être permis de mieux montrer l'intérêt du texte de Jan Baetens sur la culture et les CS : une défense bien argumentée, fondée sur une stratégie complémentariste et sur l'individuation d' " alliés » de grande envergure. Grace à des auteurs comme lui, les CS restent importants et même urgents aujourd'hui, comme contribution pour comprendre les cultures et les formes de production de sens, et comme tremplin pour se risquer dans des nouvelles formes de recherche trans- et interdisciplinaire (mais) rigoureuse.

\section{Bibliographie}

Anne Beyaert-Geslin et Jacques Fontanille (2003). « Modèles sémiotiques et textualité. Introduction ». In Modèles linguistiques, Tome XXIV, Fascicule 1.

Umberto Eco (1990/1999 ed. franc.). Les limites de l'interprétation. Paris, Lgf/Le livre de Poche.

- (1995). «Sul simbolo ». In Sandro Briosi (éd.), L'immagine riflessa, N. S. Anno IV, n. 1, Alessandria, Edizioni dell'Orso.

Jacques Fontanille (2008). Pratiques sémiotiques. Parigi, PUF.

Jacques Fontanille et Claude Zilberberg (1998). Tension et signification. Sprimont, Mardaga.

Roberto Grandi (1994). I mass media fra testo e contesto. Milano, Lupetti.

Nathalie Heinich (2002). La sociologie de l'art. Paris, La Découverte.

Christian Jouhaud (2007). Sauver le Grand Siècle? Paris, Seuil.

Jean-Marie Schaeffer (2007). La fin de l'exception humaine. Paris, Gallimard. 